\title{
ERRATUM
}

\section{Hyperglycemia decreases anti-cancer efficiency of adriamycin via AMPK pathway}

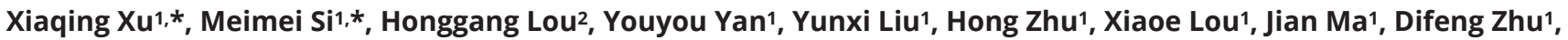
Honghai Wu' ${ }^{1}$, Bo Yanz'1 Haoshu Wu' ${ }^{1}$, Ling Ding ${ }^{1}$ and Qiaojun $\mathrm{He}^{1}$

1Zhejiang Province Key Laboratory of Anti-Cancer Drug Research, Institute of Pharmacology \& Toxicology and Biochemical Pharmaceutics, College of Pharmaceutical Sciences, Zhejiang University, Hangzhou, China

2Department of Clinical Pharmacology, The Second Affiliated Hospital, College of Medicine, Zhejiang University, Hangzhou, China

Correspondence should be addressed to L Ding or Q He: Id362@zju.edu.cn or qiaojunhe@zju.edu.cn

*(X Xu and M Si contributed equally to this work)

The authors and journal apologise for an error in the above paper, which appeared in volume 25 part 11, pages 955-966. The error relates to the artwork of Fig. 5 on page 963, in which the blots given in panel E were mistakenly replicated in panel F.

The correct Fig. 5 is given in full below: 
A
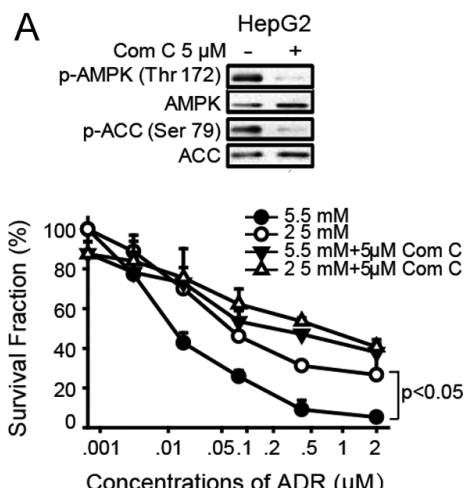

C
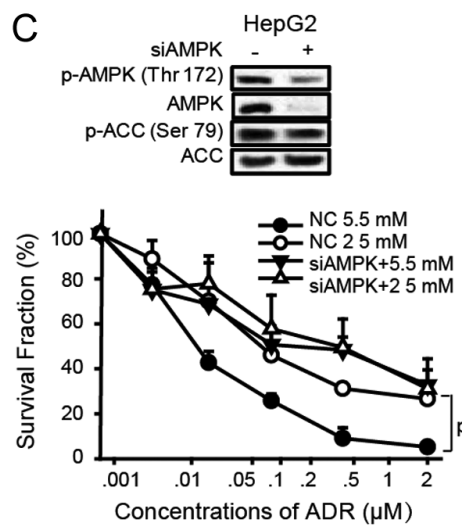

E
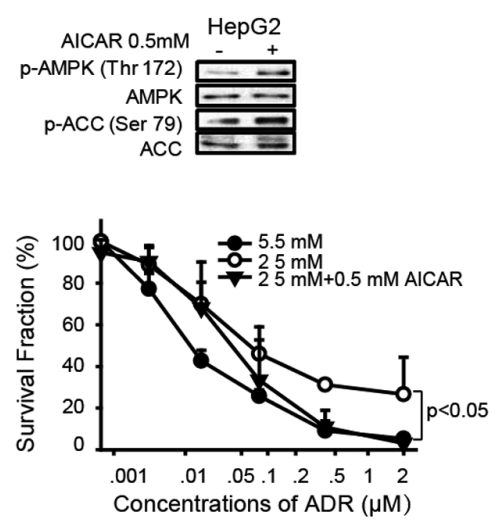

B
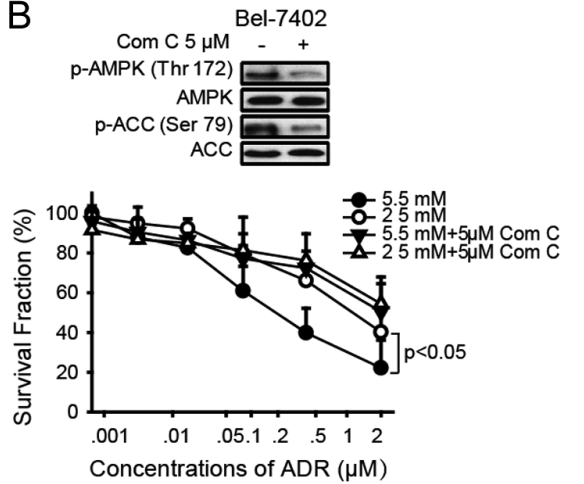

D
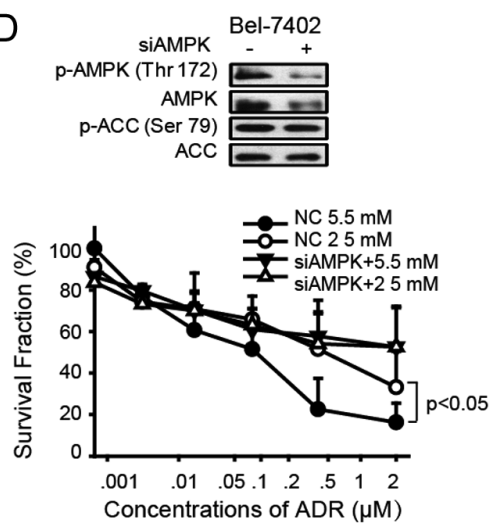

$\mathrm{F}$
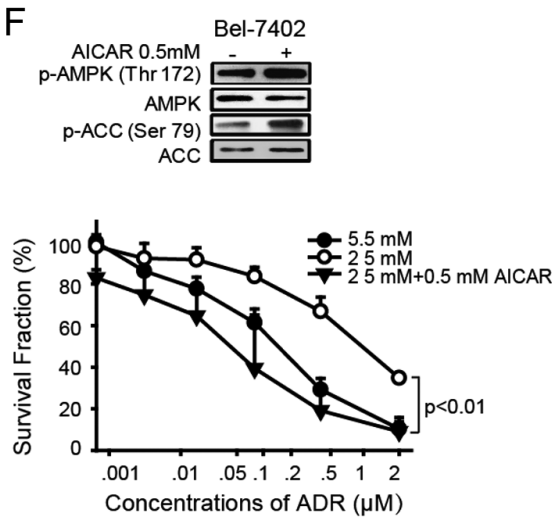

\section{Figure 5}

High glucose decreases the cytotoxicity of ADR by downregulating AMPK pathway. (A and B) HepG2 and Bel-7402 cells were pretreated with $5 \mu \mathrm{M}$ compound C for 2 h. (C and D) HepG2 and Bel-7402 cells were transfected with siRNAs specifically targeting AMPK or control siRNAs. ( $E$ and $F$ ) HepG2 and Bel-7402 cells were pretreated with $0.5 \mathrm{mM}$ AICAR for $2 \mathrm{~h}$. Then, cells were exposed to ADR as indicated in different glucose conditions for $48 \mathrm{~h}$. Cell-proliferation inhibitory activities were determined by SRB assay. Protein expressions were detected by Western blot analysis. 